\title{
Bifosfonatos e as repercussões nos maxilares: Uma revisão narrativa
}

\author{
Bisphosphonates and the jaw repercussions: A narrative review \\ Bifosfonatos y las repercusiones de las mandíbulas: Una revisión narrativa
}

Recebido: 05/07/2021 | Revisado: 11/07/2021 | Aceito: 17/07/2021 | Publicado: 26/07/2021

Michelly Cauás de Queiroz Gatis

ORCID: https://orcid.org/0000-0001-6372-3845

Universidade de Pernambuco, Brasil

E-mail: michelly.cauas@upe.br

Aldson dos Santos Silva

ORCID: https://orcid.org/0000-0003-1824-062X

Universidade Federal de Pernambuco, Brasil E-mail: aldson.silva@ufpe.br

Maria Luiza Alcoforado Ribeiro

ORCID: https://orcid.org/0000-0003-4344-1765

Universidade de Pernambuco, Brasil

E-mail: malualcoforador@gmail.com

Davi Barbirato

ORCID: https://orcid.org/0000-0003-0527-6092

Universidade de Pernambuco, Brasil

E-mail: davibarbirato@gmail.com

Elizabeth Carneiro de Arruda Ponzi

ORCID: https://orcid.org/0000-0002-2657-9343

Universidade de Pernambuco, Brasil

E-mail:bethcirurgia@yahoo.com.br

José Brasiliense Holanda Cavalcanti Filho ORCID: https://orcid.org/0000-0001-6779-7681

Universidade de Pernambuco, Brasil

E-mail:josebrasiliensehcf@yahoo.com.br

Carlos Augusto Pereira do Lago

ORCID: https://orcid.org/0000-0001-9457-714X

Universidade de Pernambuco, Brasil

E-mail:carlos.lago@upe.br

Belmiro Cavalcanti do Egito Vasconcelos

ORCID: https://orcid.org/0000-0002-6515-1489

Universidade de Pernambuco, Brasil

E-mail: belmiro.vasconcelos@upe.br

\begin{abstract}
Resumo
Os Bifosfonatos são fármacos que atuam diminuindo e inibindo a atividade osteoclástica, e induzindo a apoptose celular. Bloqueiam a diferenciação dos osteoclastos e estimulam os osteoblastos, acarretando a diminuição da reabsorção óssea. Podendo contribuir para exposição de osso necrótico na região bucomaxilofacial. Desta forma, a presente revisão narrativa propõe uma breve discussão, acerca do desenvolvimento da osteonecrose dos ossos maxilares decorrentes da terapia com bifosfonatos. Tendo como temática o desenvolvimento da osteonecrose nos maxilares decorrentes do uso dos bifosfonatos, buscou-se através dos MeSH terms e DeCS/MeSH terms, entre 2006 e 2021, artigos no idioma inglês e português indexados na base de dados do MEDLINE/PubMed, Web of Science, LILACS/bvs bem como, através de uma busca manual. Após a análise dos artigos selecionados observou-se que a possibilidade do desenvolvimento da osteonecrose nos ossos maxilares é multifatorial, sendo desta forma imperativo uma abordagem, a estes sítios, de cunho preventivo e com uma terapêutica minimamente invasiva.
\end{abstract}

Palavras-chave: Bifosfonatos; Osteonecrose; Mandíbula.

\begin{abstract}
Bisphosphonates are drugs that act by decreasing and inhibiting osteoclastic activity, and inducing cell apoptosis. They block osteoclast differentiation and stimulate osteoblasts, resulting in decreased bone resorption. It may contribute to the exposure of necrotic bone in the maxillofacial region. Thus, this narrative review proposes a brief discussion about the development of osteonecrosis of the maxillary bones resulting from therapy with bisphosphonates. Having as the theme the development of osteonecrosis in the jaws resulting from the use of bisphosphonates, we searched through the MeSH terms and DeCS/MeSH terms, between 2006 and 2021, for articles in English and Portuguese indexed in the MEDLINE/PubMed database, Web of Science, LILACS/bvs as well as
\end{abstract}


through a manual search. After analysis the selected articles, it was observed that the possibility of the development of osteonecrosis in the maxillary bones is multifactorial, thus it is imperative to approach these sites with a preventive way and with a minimally invasive therapy.

Keywords: Bisphosphonates; Osteonecrosis; Jaw.

\section{Resumen}

Los bifosfonatos son fármacos que actúan disminuyendo e inhibiendo la actividad osteoclástica e induciendo la apoptosis celular. Bloquean la diferenciación de los osteoclastos y estimulan los osteoblastos, lo que reduce la resorción ósea. Puede contribuir a la exposición de hueso necrótico en la región maxilofacial. Por lo tanto, esta revisión narrativa propone una breve discusión sobre el desarrollo de la osteonecrosis de los huesos maxilares como resultado de la terapia con bifosfonatos. Teniendo como tema el desarrollo de osteonecrosis en los maxilares como resultado del uso de bifosfonatos, buscamos a través de los términos MeSH y DeCS/MeSH, entre 2006 y 2021 , artículos en inglés y portugués indexados en la base de datos MEDLINE/PubMed, Web of Science, LILACS/bvs así como mediante búsqueda manual. Después de analizar los artículos seleccionados, se observó que la posibilidad del desarrollo de osteonecrosis en los maxilares es multifactorial, por lo que es imperativo abordar estos sitios de forma preventiva y con una terapia mínimamente invasiva.

Palabras claves: Bifosfonatos; Osteonecrosis; Mandíbula.

\section{Introdução}

Os bifosfonatos (BFs) são análogos sintéticos do pirofosfato, um regulador endógeno da mineralização óssea (Crépin et al.,2010), frequentemente usados na osteomodulação em doenças ósseas benignas, como a osteoporose, osteogênese imperfeita e doença de Paget e, em doenças malignas como o mieloma múltiplo e metástases ósseas atreladas a câncer de próstata e de mama (Granate-Marques et al., 2019). Estes fármacos atuam reduzindo a reabsorção óssea, estimulando a atividade osteoblástica e a apoptose de osteoclastos, buscando paralizar a progressão da perda óssea e promovendo o aumento da densidade (Dotto \& Dotto, 2011), bem como apresentar efeitos adversos como úlcera péptica, esofagites, falência renal, mialgia (Izquierdo et al., 2011), e osteonecrose dos ossos maxilares (Rigo et al., 2017).

Por serem agentes antirreabsortivos potentes, os bisfosfonatos representam a terapia de primeira linha para a prevenção de fraturas osteoporóticas. Os bifosfonatos ficam incrustados no novo osso na fase anabólica de remodelação, ligando-se a hidroxiapatita, permanecendo inerte. Havendo reabsorção óssea, os BPs são liberados na lacuna ácida criada pelos osteoclastos e são absorvidos pelas células, nos BPs que não apresentam nitrogênio induzem a apoptose do osteoclasto ao se ligar ao ATP (Kates \& Ackert-Bicknell, 2016); e os que possuem nitrogênio inibem a farnesil pirosfosfato sintase, enzima chave na via do mevalonato (Chapurlat, \& Delmas, 2006).

O desenvolvimento da osteonecrose dos maxilares, caracterizada por uma área de exposição óssea que não se repara em oito semanas (Khosla et al., 2007), acomete pacientes expostos ao uso de BFs sistêmico e, pacientes não submetidos a radioterapia no complexo maxilomandibular (Costa et al., 2019; Sales \& Conceição, 2020).

Quanto a casuística de osteonecrose induzida por bisfosfonatos, os estudos demonstram que pode haver uma prevalência relacionado ao tempo e via de administração (Devogelaer et al., 2007; Lyles et al., 2007; Grbic et al., 2008; Etminan et al., 2008; Pazianas, 2008; Coelho, 2010; Khan et al., 2011; Powel et al., 2012; Tennis et al., 2012; Ulmner et al., 2014 De Moraes, 2014) tipo de droga administrada ou mesmo associação de fármacos (Tavares Junior et al., 2006; Izquierdo et al., 2011; Brozoski et al., 2012; Fliefel et al., 2015; Costa et al., 2019), assim demonstrado por Srivastava et al., (2021) na revisão sistemática sobre qual a prevalência de osteonecrose da mandíbula e bifosfonatos, onde revelaram uma prevalência ponderada de 13\% (IC 95\% 3-22\%) para terapia sequencial com bifosfonato-denosumabe, apenas bifosfonatos foi de 5\% (IC 95\% 0-9\%) e denosumabe de $4 \%$ (IC 95\% 3- 5\%).

Assim, diante da possibilidade de ocorrência desta enfermidade, da necessidade de uma abordagem curativa prévia (Brozoski, 2012), bem como um suporte preventivo na vigência da mesma (Aparecida et al., 2018), a presente revisão narrativa 
propõe uma breve discussão, acerca do desenvolvimento da osteonecrose dos ossos maxilares decorrentes da terapia com bifosfonatos.

\section{Metodologia}

Este estudo, desenvolvido a partir de conceitos de uma revisão narrativa qualitativa, baseia-se em descrever e discutir um tema, levando em consideração uma base teórica ou contextual (Rother, 2007). As estratégias de busca foram estruturadas a partir de MeSH terms e DeCS/MeSH terms e pesquisadas nas bases de dados MEDLINE/PubMed, Web of Science e LILACS/bvs na busca por artigos publicados entre 2006 e 2021, nos idiomas inglês e português. A mesma estratégia de busca foi aplicada na busca manual (Tabela 1).

\section{Resultados e Discussão}

Foram incluídos artigos que abordassem o uso de bifosfonatos e a consequente ocorrência de osteonecrose nos ossos maxilares, excluindo aqueles realizados em animais, in vitro, assim como cartas ao editor, textos de opiniões e os que não fosse possível acessar na íntegra.

A busca inicial recuperou 730 artigos. Destes, após a leitura de título e resumo, foram excluídos 640 estudos; no total, foram selecionados 90 artigos para inclusão na revisão, a partir dos critérios de elegibilidade. Dois revisores (EACP e MCQG) participaram da avaliação dos resumos dos artigos selecionados de forma independente (mascaramento) e apenas os estudos selecionados por ambos revisores foram incluídos.

Tabela 1: MeSH terms/ e DeCS/MeSH terms.

\begin{tabular}{|l|}
\hline MEDLINE/PubMed, Web of Science \\
\hline ("bisphosphonate associated osteonecrosis of the jaw"[MeSH Terms] OR ("bisphosphonate associated"[All \\
Fields] AND "osteonecrosis"[All Fields] AND "jaw"[All Fields]) OR "bisphosphonate associated osteonecrosis of \\
the jaw"[All Fields] OR ("bisphosphonate"[All Fields] AND "associated"[All Fields] AND "osteonecrosis"[All \\
Fields] AND "jaw"[All Fields]) OR "bisphosphonate associated osteonecrosis of the jaw"[All Fields]) AND \\
((clinicaltrial[Filter] OR meta-analysis[Filter] OR randomizedcontrolledtrial[Filter] OR review[Filter]) AND \\
(2006/1/1:2021/6/21[pdat])) \\
\hline LILACS/bvs; busca manual \\
(osteonecrose associada a bifosfonatos) OR (osteonecrose associada a bifosfonatos) OR (osteonecrose associada \\
difosfonatos ) OR (osteonecrose associada aos bifosfonatos ) OR (osteonecrose associada aos bifosfonatos ) OP \\
(osteonecrose associada aos difosfonatos) OR (osteonecrose da arcada osseodentária associada a bifosfonatos ) OP \\
(osteonecrose da arcada osseodentária associada a bifosfonatos ) OR ( osteonecrose da arcada osseodentári \\
associada aos bifosfonatos ) OR (osteonecrose da arcada osseodentária associada aos bifosfonatos ) OH \\
(osteonecrose da arcada osseodentária por bifosfonatos ) OR (osteonecrose da arcada osseodentária po \\
bifosfonatos OR osteonecrose da arcada osseodentária por difosfonatos) AND (fulltext:("1" OR "1") AND la:("en \\
OR "pt")) AND (year_cluster:[2006 TO 2021]) \\
\hline
\end{tabular}

Fonte: Autores.

Os bisfosfonatos (BFs) diminuem a reabsorção óssea, inibindo a atividade e o recrutamento dos osteoclastos, promovendo a apoptose e podendo interferir na cicatrização óssea (Chang et al., 2018) farmacologicamente apresenta variados níveis de potência e presença ou não de base nitrogenada. Podem ser indicados para tratamento de patologias ósseas como a doença de Paget, osteogênese imperfeita e osteoporose pós-menopausa (Poubel et al., 2012).

Estes fármacos possuem alta afinidade pela hidroxiapatita na matriz óssea e permanecem integrados à matriz por cerca de 10 anos, devido a sua estrutura química. Quando os osteoclastos aderem à superfície óssea para iniciar a reabsorção, liberam 
enzimas que acidificam o ambiente e promovem a degradação óssea. Estes degradados ricos em BFs são fagocitados pelos osteoclastos inibindo a reabsorção óssea pela inativação da via da síntese do colesterol dentro da célula, reduz a atividade celular ou promove a apoptose, interrompendo assim, o ciclo dos osteoclastos (Querrer et al., 2021; Papapoulos, 2008).

Como o suprimento sanguíneo para a região cortical é derivado do periósteo, a exposição óssea é indicativa de osso necrótico subjacente, que pode evoluir com quadro flogistico de diferentes gradações culminando ou não em fraturas patológicas, presença de condensação óssea na região de lâmina dura e/ou crista milo-hioidea, além de alargamento dos ligamentos periodontais (Khan et al., 2009).

A osteonecrose é uma terminologia que se refere à morte celular de dois componentes do osso, a medula hematopoiética e os osteócitos. A osteonecrose de origem medicamentosa apresenta uma frequência de 8:1 para homens em relação às mulheres, em idade inferior a 50 anos (Borjaille et al., 2006); esta patologia é pouco frequente ou menos observada em crianças (Christou et al., 2013). Para ser classificada como osteonecrose dos ossos maxilares associada ao uso de bifosfonatos (ONMAB), atualmente descrita como osteonecrose medicamentosa, deve preencher os seguintes critérios: (1) tratamento atual ou anterior com agentes anti-reabsortivos ou antiangiogênicos (Khan et al., 2015); (2) osso exposto ou osso que pode ser sondado através de uma fístula intraoral ou extra oral na região maxilofacial que persistiu por mais de 8 semanas; e (3) nenhuma história de radioterapia nos maxilares ou doença metastática óbvia nos maxilares (Ruggiero et al., 2014; Frascino \& Forte, 2016; Rigo et al., 2017).

Os fatores de risco são divididos em:

1. Fatores sistêmicos; que incluem tipo de BFs, a indicação terapêutica, sexo, idade, fatores genéticos (Kalra \& Jain, 2013) e comorbidades como a artrite reumatoide (Duarte, et al.,2019).

2. Fatores ambientais: via de administração, duração do tratamento, dose cumulativa, terapias concomitantes, como agentes antiagiogênicos; fumo, álcool (Sarasquete et al., 2009).

3. Fatores de risco locais, como cirurgia dentoalveolar, lesão periapical (AlRahabi, \& Ghabbani, 2018) e periodontal e uso de próteses removíveis; e

4. Fatores anatômicos, como o tórus mandibular (Tsao et al., 2013; Rasmusson \& Abtahi, 2014; Gavalda \& Bagan, 2016).

Apesar de ainda não se ter uma total compreensão do mecanismo de ação dos bifosfonatos (Hagelauer et al.,2014), é possível que esteja atrelado à inibição da função dos osteoclastos e consequente diminuição da capacidade de remodelação óssea, impedimento da atividade angiogênica (Otto et al.,2013). Pois, variações genéticas (Guo et al.,2020) e diminuição do suprimento vascular, relacionados com a isquemia, também podem levar à necrose tecidual (Santos et al., 2011; Barinet al., 2016).

A topografia mandibular e maxilar, pode chegar a uma proporção de 2:1, respectivamente, (Cunha et al.,2019) para o apareciemento da osteonecrose, que pode ser justificado por fatores como: a atividade funcional diária e processos de remodelação óssea (turnover) (Frascino \& Forte, 2016), próximo ao ligamento periodontal, local em que os bifosfonatos apresentam afinidade (Dotto \& Dotto, 2011), e possibilidade de indução das células T, produtoras de RANKL - ativadores dos osteoclastos (Walter et al.,2013).

Características funcionais do colágeno, incluindo uma maior quantidade de colágeno, uma menor quantidade de reticulações maduras e uma menor extensão de hidroxilação de Lys, que exigem uma alta taxa de renovação para o funcionamento da mecânica mandibular e interação celular, também podem contribuir para a osteonecrose medicamentosa (Matsuura et al., 2014). 
Outro ponto a ser considerado para esta preferencia topográfica é quanto a presença ou não de uma base nitrogenada, como pontuado por Endo (2017):

1. N-BPs se acumulam nos ossos maxilares após repetida administração e esse acúmulo é promovido pela inflamação causada por bactérias orais e/ou extração dentária;

2. $\quad$ N-BPs acumulados são liberados pela destruição óssea causada por infecção e/ou extração dentária

3. TLR4 (receptor do tipo Toll 4) é regulado positivamente pelos N-BPs;

4. $\quad$ N-BPs também são liberados pelos osteoclastos mortos;

5. N-BPs (administrados e liberados dos ossos) são levados para as células dos tecidos moles em torno do osso da mandíbular, via transportadores de fosfato e os N-BPs introduzidos nas células dos tecidos moles induzem inflamação e/ou morte celular, devido à sua citotoxicidade. Podendo acarrestar um ciclo vicioso e assim disseminar a necrose através dos maxilares e exposição óssea. (Sawatari \& Marx, 2007; Ruggiero \& Woo, 2008; Mariotti, 2008).

Os achados histológicos mais comuns incluem regiões de osso inviável, mostrando perda de osteócitos, reabsorção periférica (Biasotto et al.,2006) com restos bacterianos circundantes e infiltração de células inflamatórias. A osteomielite aguda ou crônica (Allen \& Burr, 2008), em que o osso necrótico apresenta centros ósseos de Havers vazios, e a presença de biofilme bacteriano complexo (Reich et al., 2015), bem como células malignas (Sacco et al.,2021), também podem estar presentes.

As características imagenológicas podem diferir a depender da droga empregada (Querrer et al., 2021) e do grau de compromentimento clínico (Kim et al.,2020). O acompanhamento imagenológico é recomendado (Moreno-Rabié, et al., 2020) desde antes do início terapêutico, sendo possível observar espessamento da lâmina dura (Cardoso et al.,2017), osteoesclerose (Arce et al., 2009), falha da remodelação óssea (Moinzadeh et al., 2013), aumento do espaço do ligamento periodontal e da densidade trabecular, cicatrização deficiente de alvéolos pós-extração, formação de sequestros ósseos, alargamento do canal mandibular e/ou do assoalho do seio maxilar, fistulas oroantrais, fístulas oronasais (Wasserzug et al., 2017) e neoformação óssea periosteal (Brozoski et al., 2012; Fliefel et al., 2015).

Imagens multimodais como a radiografia panorâmica, cintilografia, tomografia computadorizada com multidetectores, imagem por ressonância magnética e tomografia computadorizada por emissão de fóton único, são úteis para detectar ONMAB. A tomografia computadorizada de feixe cônico fornece detalhes anatômicos precisos como textura interna lítica e esclerótica, presença de sequestro e reação periosteal vestibular e lingual e perfuração cortical (Ogura et al.,2021).

Clinicamente, o osso exposto e necrótico pode permanecer assintomático por semanas, meses ou até anos (Allen et al., 2009), ou pode ocorrer a percepção dolorosa relacionada à inflamação dos tecidos circundantes ou não (Srinvasan, et al.,2007), associada ou não com diminuição do fluxo salivar e mobilidade dentária (Stockmann, et al.,2020). Os sintomas podem ocorrer espontaneamente, antes do desenvolvimento de osteonecrose clinicamente detectável (Otto et al., 2009; Fedele et al., 2010; Zadik et al., 2012; Sharma et al., 2013).

O estágio clínico da osteonecrose mandibular por bifosfonatos (Tabela 02) fornece um guia para a instituição de uma proposta terapêutica (Ruggiero \& Woo, 2009). O manejo permeia do sintomático, com a utilização de analgésicos e antibióticos, - Estágio 0- até a necessidade de desbridamento e ressecção cirúrgica no Estágio 3 se contudo, ter um protocolo fechado (Brozoski et al., 2012) e sim possibilidades terapêuticas que melhorem o quadro clínico e qualidade de vida do paciente (Passeri et al., 2011; Leite et al., 2015; Fliefel et al., 2015; Bodem et al., 2016). 
Tabela 2: O estágio clínico da osteonecrose mandibular por bifosfonatos.

\begin{tabular}{|c|l|}
\hline ESTÁGIO & \multicolumn{1}{c|}{ APRESENTAÇÃO CLÍNICA } \\
\hline EM RISCO & Sem exposição óssea Orientações ao paciente \\
\hline 1 & $\begin{array}{l}\text { Exposição óssea assintomática com pequena inflamação de tecido } \\
\text { mole. Proposta: Orientações ao paciente, bochechos com soluções } \\
\text { antibacaterianas e acompanhamento criterioso. }\end{array}$ \\
\hline 2 & $\begin{array}{l}\text { Osso exposto com dor, inflamação ou infecção de tecido mole } \\
\text { adjacente. Proposta: Orientações ao paciente, bochechos com } \\
\text { soluções antibacterianas, antibioticoterapia, desbridamento } \\
\text { superficial e acompanhamento criterioso }\end{array}$ \\
\hline 3 & $\begin{array}{l}\text { Osso exposto com dor, inflamação ou infecção de tecido mole } \\
\text { adjacente, podendo apresentar osteólise estendedo-se até a borda } \\
\text { inferior da mandíbula ou fraturas patológicas e fístulas extraoral. } \\
\text { Proposta: Orientações ao paciente, bochechos com soluções } \\
\text { antibacterianas, cirurgias palativas e acompanhamento criterioso. }\end{array}$ \\
\hline
\end{tabular}

Fonte: Ruggiero e Woo (2009).

Assim, devido a possibilidade de o paciente evoluir com ONMAB, é importante avaliar e realizar tratamentos odontológicos definitivos (Souza Junior et al.,2020) antes de iniciar terapias com bifosfonatos (De Souza et al., 2018), como uma estratégia preventiva. Uma vez instalada a osteonecrose medicamentosa, os procedimentos ou as técnicas minimamente invasivas devem ser prioridade no tratamento odontológico (De Moraes et al., 2014; Junior et al., 2016; De Souza et al., 2018; Papadakis et al.,2021), e, cabe orientar o paciente quanto à importância de se manter a higiene oral adequada (Coelho et al., 2010; Izquierdo et al., 2011; Ferreira et al.,2021). O cuidado com traumas locais e o uso adequado de agentes antimicrobianos bucais podem reduzir a incidência de ONMAB (Thumbigere-Math et al., 2014).

Procedimentos odontológicos mais comuns e invasivos como extrações dentárias (Souza Junior et al., 2020) e instalação de implantes dentários (Leite et al., 2015; Santos et al., 2016) devem ser planejados e executados de forma criteriosa e ponderar a real necessidade de sua realização (Chaves et al., 2018; Stramandinoli-Zanicotti et al., 2018), especialmente antes de iniciar o tratamento com bisfosfonatos. Quando sua execução for imperativa, exames laboratoriais pré-operatórios como a dosagem do Telopeptídeo-C Terminal ou colágeno tipo I (CTX), marcadores específicos da reabsorção óssea liberados durante a remodelação e renovação óssea, podem auxiliar no planejamento e sugerir risco de ocorrência de osteonecrose pósoepratória. Esta possibilidade se baseia no fato de os bifosfonatos reduzirem o CTX após 3 meses de terapia. Tendo como parametro de normalidade valores normais do CTX de 300-600 pg/ml, os valores inferiores a $100 \mathrm{pg} / \mathrm{ml}$ representam alto risco para osteonecrose, entre 100 e $150 \mathrm{pg} / \mathrm{ml}$ risco representam risco moderado e acima de $150 \mathrm{pg} / \mathrm{ml}$ representam baixo risco de ocorrência de osteonecrose, indicada para planejamentos cirúrgicos de pacientes que fizeram uso ou fazem uso de bisfosfonatos (De Moraes et al., 2014; Chaves et al., 2018).

A possibilidade de suspender o uso de BFs é questionada (Chaves et al., 2018) em razão da sua permanência na matriz óssea por longos períodos. Entretanto, a literatura sugere que nos três meses que antecedem o procedimento cirúrgico e por três meses após o mesmo, essa suspensão pode favorecer a cicatrização, salientado que há um melhor prognóstico para os BFs administrados por via oral (De Oliveira et al., 2014). Vilela-Carvalho (2018) sugeriram uma suspensão de uso da droga 2 meses antes da realização de procedimentos cirúrgicos para pacientes em tratamento recente e por via de administração oral, e, 3 meses para pacientes expostos à droga por um período prolongado ( $>4$ anos). Contudo, ainda não existe um consenso sobre a suspensão de uso dos BFs e o risco de osteonecrose medicamentosa dos maxilares, especialmente para pacientes em tratamento por muitos anos ou com drogas endovenosas. Portanto, as abordagens terapêutica e preventiva devem sempre ser planejadas e executadas desde antes do início do tratamento com BFs, ponderando-se os riscos e benefícios de cada 
panejamento odontológico possível para uma futura ocorrência de osteonecrose medicamentosa ao longo dos anos (Coelho et al., 2010).

\section{Conclusão}

A possibilidade do desenvolvimento da osteonecrose nos ossos maxilares é multifatorial, sendo desta forma imperativo uma abordagem, a estes sítios, de cunho preventivo e com uma terapêutica minimamente invasiva.

\section{Referências}

Allen, M. R., \& Burr, D. B. (2008). Mandible matrix necrosis in beagle dogs after 3 years of daily oral bisphosphonate treatment. Journal of oral and maxillofacial surgery: official journal of the American Association of Oral and Maxillofacial Surgeons, 66(5), 987-994. https://doi.org/10.1016/j.joms.2008.01.038

AlRahabi, M. K., \& Ghabbani, H. M. (2018). Clinical impact of bisphosphonates in root canal therapy. Saudi medical journal, 39(3), 232-238. https://doi.org/10.15537/smj.2018.3.20923

Aparecida Cariolatto, F., Carelli, J., de Campos Moreira, T., Pietrobon, R., Rodrigues, C., \& Bonilauri Ferreira, A. P. (2018). Recommendations for the Prevention of Bisphosphonate-Related Osteonecrosis of the Jaw: A Systematic Review. The journal of evidence-based dental practice, 18(2), 142-152. https://doi.org/10.1016/j.jebdp.2017.11.002

Arce, K., Assael, L. A., Weissman, J. L., \& Markiewicz, M. R. (2009). Imaging findings in bisphosphonate-related osteonecrosis of jaws. Journal of oral and maxillofacial surgery: official journal of the American Association of Oral and Maxillofacial Surgeons, 67(5 Suppl), 75-84. https://doi.org/10.1016/j.joms.2008.12.002

Barin, L. M., Pillusky, F. M., Pasini, M. M., Denesi, C. C. (2016) Osteonecrose dos maxilares associada ao uso de bifosfonatos: uma revisão de literatura. Revista de Odontologia da Universidade Cidade de São Paulo, 28(2), 126-134. ISSN 1983-5183

Biasotto, M., Chiandussi, S., Dore, F., Rinaldi, A., Rizzardi, C., Cavalli, F., \& Di Lenarda, R. (2006). Clinical aspects and management of bisphosphonatesassociated osteonecrosis of the jaws. Acta odontologica Scandinavica, 64(6), 348-354. https://doi.org/10.1080/00016350600844360

Bodem, J. P., Schaal, C., Kargus, S., Saure, D., Mertens, C., Engel, M., Hoffmann, J., \& Freudlsperger, C. (2016). Surgical management of bisphosphonaterelated osteonecrosis of the jaw stages II and III. Oral surgery, oral medicine, oral pathology and oral radiology, 121(4), 367-372. https://doi.org/10.1016/j.oooo.2015.10.033

Borjaille, B.P., Brandão, L.R., Hasegawa, T.M., Rosa, R. F., Antonio, S.F., Chahade, W. H., (2006). Osteonecrose e Síndrome da Imunodeficiência Adquirida. Rev. Bras. Reumatol, 46, suppl.1,36-44.

Brozoski, M. A., Traina, A. A., Deboni, M. C. Z., Marques, M. M., Naclério-Homem, M. da G. (2012). Osteonecrose maxilar associada ao uso de bifosfonatos. Rev Bras Reumatol 2012;52(2):260-270.

Cardoso, C. L., Barros, C. A., Curra, C., Fernandes, L. M. P. da S. R., Franzolin, S. de O. B., Júnior, J. S. F., Curi, M. M. (2017). Radiographic Findings in Patients with Medication-Related Osteonecrosis of the Jaw. International Journal of Dentistry, 2017, 1-6. doi:10.1155/2017/3190301

Chang, J., Hakam, A. E., \& McCauley, L. K. (2018). Current Understanding of the Pathophysiology of Osteonecrosis of the Jaw. Current osteoporosis reports, 16(5), 584-595. https://doi.org/10.1007/s11914-018-0474-4

Chapurlat, R. D., \& Delmas, P. D. (2006). Drug insight: Bisphosphonates for postmenopausal osteoporosis. Nature clinical practice. Endocrinology \& metabolism, 2(4), 211-238. https://doi.org/10.1038/ncpendmet0121

Chaves, R. A. da C., Órfão, A. M. A., Júnior, W. B., Queiroz, T. P., \& Faloni, A. P. (2018). Bifosfonatos e Denosumabes: mecanismos de ação e algumas implicações para a implantodontia. Revista Brasileira Multidisciplinar, 21(2), 66-80. https://doi.org/10.25061/2527-2675/ReBraM/2018.v21i2.483

Christou, J., Johnson, A. R., \& Hodgson, T. A. (2013). Bisphosphonate-related osteonecrosis of the jaws and its relevance to children--a review. International journal of paediatric dentistry, 23(5), 330-337. https://doi.org/10.1111/ipd.12047

Coelho, A. I., Gomes, P. De S., Fernandes, M. H. (2010) Osteonecrose dos maxilares associada ao uso de bifosfonatos. Parte I: etiologia e apresentação clínica. Revista Portuguesa de Estomatologia, Medicina Dentária eCirurgia Maxilofacial, 51(2), 95-101. https://doi.org/10.1016/S1646-2890(10)70093-5

Costa, L. L. R., Mendes, T. M., Santos, L. S., Mendes, P. H. C. (2019). Análise do conhecimento e da conduta médica acerca da prevenção da osteonecorose dos maxilares pelo uso de bifosfonatos. Revista da Universidade Vale do Rio Verde, 17(1),1-8. http://dx.doi.org/10.5892/ruvrd.v17i1.4981

Crépin, S., Laroche, M. L., Sarry, B., \& Merle, L. (2010). Osteonecrosis of the jaw induced by clodronate, an alkylbiphosphonate: case report and literature review. European journal of clinical pharmacology, 66(6), 547-554. https://doi.org/10.1007/s00228-010-0822-5

Cunha, K. S., Lippert, V. F., Baldino, M. E. L., Dreyer, J. W., Cunha, R. M. (2019) Alternativa de tratamento reabilitador para pacientes emuso de bisfosfonato intravenoso: relato de caso clinico. Revista Odontológica de Araçatuba, 40(1), 29-34. ISSN 2357-8378 e $1677-6704$

De Moraes, S. L. C., Afonso, A. M. P., Santos, R. G. S., Mattos, R. P., Oliveira, M. T. F., Zanetta-Barbosa, D., Duarte, B. G. (2014) Riscos e complicações para os ossos da face decorrentes do uso de bifosfonatos. Revista Brasileira de Odontologia, 70(2),114-119. ISSN 1984-3747. 
De Oliveira, M. A., Martins, M. F., Asah, D. A., Santos, P. S., Gallotini, M. (2014) Osteonecrose induzida por Bifosfonatos: relatode caso clínico e protocolo de atendimento/Osteonecrosis induced by bisphosphonates: case study and protocol service. Arquivos Médicos dos Hospitais e da Faculdade de Ciências Médicas da Santa Casa de São Paulo, 59(1), 43-48.

De Sousa, A. S., Taitra, V. P. A. J., Saveda, L. F., Rodrigues, I. V., Giro, G. (2018) Protocolo de atendimento odontológico de pacientes em tratamento com bifosfonatos. Revista Saúde-UNG-Ser, 12(1/2,), 54-61. http://dx.doi.org/10.33947/1982-3282-v12n1-2-3278

Devogelaer, J. P., Brown, J. P., Burckhardt, P., Meunier, P. J., Goemaere, S., Lippuner, K., Body, J. J., Samsioe, G., Felsenberg, D., Fashola, T., Sanna, L., Ortmann, C. E., Trechsel, U., Krasnow, J., Eriksen, E. F., \& Garnero, P. (2007). Zoledronic acid efficacy and safety over five years in postmenopausal osteoporosis. Osteoporosis international: a journal established as result of cooperation between the European Foundation for Osteoporosis and the National Osteoporosis Foundation of the USA, 18(9), 1211-1218. https://doi.org/10.1007/s00198-007-0367-3

Dotto, A. C.\& Dotto, A. C. (2011) Osteonecrose dos maxilares induzida por Bifosfonatos-revisão de literatura e relato de caso. Revista da Faculdade de Odontologia-UPF,16(2),229-233.

Duarte, N. T., Rech, B. de O., Martins, I. G., Franco, J. B., \& Ortega, K. L. (2019). Can children be affected by bisphosphonate-related osteonecrosis of the jaw? A systematic review. International Journal of Oral and Maxillofacial Surgery. doi:10.1016/j.ijom.2019.08.004

Endo, Y., Kumamoto, H., Nakamura, M., Sugawara, S., Takano-Yamamoto, T., Sasaki, K., \& Takahashi, T. (2017). Underlying Mechanisms and Therapeutic Strategies for Bisphosphonate-Related Osteonecrosis of the Jaw (BRONJ). Biological \& pharmaceutical bulletin, 40(6), 739-750. https://doi.org/10.1248/bpb.b16-01020

Etminan, M., Aminzadeh, K., Matthew, I. R., \& Brophy, J. M. (2008). Use of oral bisphosphonates and the risk of aseptic osteonecrosis: a nested case-control study. The Journal of rheumatology, 35(4), 691-695.

Fedele, S., Porter, S. R., D'Aiuto, F., Aljohani, S., Vescovi, P., Manfredi, M., Arduino, P. G., Broccoletti, R., Musciotto, A., Di Fede, O., Lazarovici, T. S., Campisi, G., \& Yarom, N. (2010). Nonexposed variant of bisphosphonate-associated osteonecrosis of the jaw: a case series. The American journal of medicine, 123(11), 1060-1064. https://doi.org/10.1016/j.amjmed.2010.04.033

Ferreira Jr, L. H., Jr, Mendonça Jr, K. D., Jr, Chaves de Souza, J., Soares Dos Reis, D. C., do Carmo Faleiros Veloso Guedes, C., de Souza Castro Filice, L., Bruzadelli Macedo, S., \& Soares Rocha, F. (2021). Bisphosphonate-associated osteonecrosis of the jaw. Minerva dental and oral science, 70(1), 49-57. https://doi.org/10.23736/S0026-4970.20.04306-X

Fliefel, R., Tröltzsch, M., Kühnisch, J., Ehrenfeld, M., \& Otto, S. (2015). Treatment strategies and outcomes of bisphosphonate-related osteonecrosis of the jaw (BRONJ) with characterization of patients: a systematic review. International journal of oral and maxillofacial surgery, 44(5), 568-585. https://doi.org/10.1016/j.ijom.2015.01.026

Frascino, A., \& Forte, A. (2016). Interação dos Bifosfonatos na Cirurgia Odontológica. Atas de Ciências da Saúde, 4(1), 12-22. http://revistaseletronicas.fmu.br/index.php/ACIS/article/view/1069

Gavalda, C., \& Bagan, J. (2016). Concept, diagnosis and classification of bisphosphonate-associated osteonecrosis of the jaws. A review of the literature. Medicina Oral Patología Oral y Cirugia Bucal, e260- e270. doi:10.4317/medoral.21001

Granate-Marques, A., Polis-Yanes, C., Seminario-Amez, M., Jané-Salas, E., \& López-López, J. (2019). Medication-related osteonecrosis of the jaw associated with implant and regenerative treatments: Systematic review. Medicina oral, patologia oral y cirugia bucal,24(2), e195-e203. https://doi.org/10.4317/medoral.22691

Grbic, J. T., Landesberg, R., Lin, S. Q., Mesenbrink, P., Reid, I. R., Leung, P. C., Casas, N., Recknor, C. P., Hua, Y., Delmas, P. D., Eriksen, E. F., \& Health Outcomes and Reduced Incidence with Zoledronic Acid Once Yearly Pivotal Fracture Trial Research Group (2008). Incidence of osteonecrosis of the jaw in women with postmenopausal osteoporosis in the health outcomes and reduced incidence with zoledronic acid once yearly pivotal fracture trial. Journal of the American Dental Association (1939), 139(1), 32-40. https://doi.org/10.14219/jada.archive.2008.0017

Guo, Z., Cui, W., Que, L., Li, C., Tang, X., \& Liu, J. (2020). Pharmacogenetics of medication-related osteonecrosis of the jaw: a systematic review and metaanalysis. International journal of oral and maxillofacial surgery, 49(3), 298-309. https://doi.org/10.1016/j.ijom.2019.07.016

Hagelauer, N., Pabst, A. M., Ziebart, T., Ulbrich, H., \& Walter, C. (2014). In vitro effects of bisphosphonates on chemotaxis, phagocytosis, and oxidative burst of neutrophil granulocytes. Clinical Oral Investigations, 19(1), 139-148. 10.1007/s00784-014-1219-0

He, L., Sun, X., Liu, Z., Qiu, Y., \& Niu, Y. (2020). Pathogenesis and multidisciplinary management of medication-related osteonecrosis of the jaw. International journal of oral science, 12(1), 30. https://doi.org/10.1038/s41368-020-00093-2

Izquierdo, C. M., Oliveira, M. G., Weber, B. B. J. (2011) Terapêutica com Bifosfonatos: implicações no paciente odontológico: revisão de literatura. RFO $U P F 16(3), 347-352$.

Junior, C. D. F., Casado, P. L., Barboza, E. S. P. (2007) Osteonecrose associada aos bifosfonatos na odontologia. Periodontia ; 17(4): 24-30.

Junior, H. H. T., Almeida, J. S., Mourão, C. F., Meira, R., Ribeiro, J. (2016) Avaliação qualitativa do tratamento da osteonecrose dos maxilares associada aos bifosfonatos: aspectos atuais da literatura. Ciência Atual-Revista Científica Multidisciplinar do Centro Universitário São José, 8(2),02-11.

Kalra, S., \& Jain, V. (2013). Dental complications and management of patients on bisphosphonate therapy: A review article. Journal of oral biology and craniofacial research, 3(1), 25-30. https://doi.org/10.1016/j.jobcr.2012.11.001

Kates, S. L., \& Ackert-Bicknell, C. L. (2016). How do bisphosphonates affect fracture healing?. Injury, 47 Suppl 1(0 1), S65-S68. https://doi.org/10.1016/S0020-1383(16)30015-8

Khan, A. A., Morrison, A., Hanley, D. A., Felsenberg, D., McCauley, L. K., O'Ryan, F., Reid, I. R., Ruggiero, S. L., Taguchi, A., Tetradis, S., Watts, N. B., Brandi, M. L., Peters, E., Guise, T., Eastell, R., Cheung, A. M., Morin, S. N., Masri, B., Cooper, C., Morgan, S. L., International Task Force on Osteonecrosis 
of the Jaw (2015). Diagnosis and management of osteonecrosis of the jaw: a systematic review and international consensus. Journal of bone and mineral research: the official journal of the American Society for Bone and Mineral Research, 30(1), 3-23. https://doi.org/10.1002/jbmr.2405

Khan, A. A., Rios, L. P., Sándor, G. K., Khan, N., Peters, E., Rahman, M. O., Clokie, C. M., Dore, E., \& Dubois, S. (2011). Bisphosphonate-associated osteonecrosis of the jaw in Ontario: a survey of oral and maxillofacial surgeons. The Journal of rheumatology, 38(7), 1396-1402. https://doi.org/10.3899/jrheum.100221

Khan, A. A., Sándor, G. K., Dore, E., Morrison, A. D., Alsahli, M., Amin, F., Peters, E., Hanley, D. A., Chaudry, S. R., Lentle, B., Dempster, D. W., Glorieux, F. H., Neville, A. J., Talwar, R. M., Clokie, C. M., Mardini, M. A., Paul, T., Khosla, S., Josse, R. G., Sutherland, S., ... Canadian Taskforce on Osteonecrosis of the Jaw (2009). Bisphosphonate associated osteonecrosis of the jaw. The Journal of rheumatology, 36(3), 478-490. https://doi.org/10.3899/jrheum.080759

Khosla, S., Burr, D., Cauley, J., Dempster, D. W., Ebeling, P. R., Felsenberg, D., Gagel, R. F., Gilsanz, V., Guise, T., Koka, S., McCauley, L. K., McGowan, J., McKee, M. D., Mohla, S., Pendrys, D. G., Raisz, L. G., Ruggiero, S. L., Shafer, D. M., Shum, L., Silverman, S. L., ... American Society for Bone and Mineral Research (2007). Bisphosphonate-associated osteonecrosis of the jaw: report of a task force of the American Society for Bone and Mineral Research. Journal of bone and mineral research: the official journal of the American Society for Bone and Mineral Research, 22(10), 1479-1491. https://doi.org/10.1359/jbmr.0707onj

Kim, J. E., Yoo, S., \& Choi, S. C. (2020). Several issues regarding the diagnostic imaging of medication-related osteonecrosis of the jaw. Imaging science in dentistry, 50(4), 273-279. https://doi.org/10.5624/isd.2020.50.4.273

Leite, G. B., Louro, R., Vivacqua, C. F. P. P., Motta, S. H. G. (2015) Osteonecrose induzida pelo uso de Bifosfonatos após instalação de implantes dentáriosrelato de caso. ImplantNews,12(6): 771-778.

Lyles, K. W., Colón-Emeric, C. S., Magaziner, J. S., Adachi, J. D., Pieper, C. F., Mautalen, C., Hyldstrup, L., Recknor, C., Nordsletten, L., Moore, K. A., Lavecchia, C., Zhang, J., Mesenbrink, P., Hodgson, P. K., Abrams, K., Orloff, J. J., Horowitz, Z., Eriksen, E. F., Boonen, S., \& HORIZON Recurrent Fracture Trial (2007). Zoledronic acid and clinical fractures and mortality after hip fracture. The New England journal of medicine, 357(18), 1799-1809. https://doi.org/10.1056/NEJMoa074941

Mariotti A. (2008). Bisphosphonates and osteonecrosis of the jaws. Journal of dental education, 72(8), 919-929.

Matsuura, T., Tokutomi, K., Sasaki, M., Katafuchi, M., Mizumachi, E., \& Sato, H. (2014). Distinct characteristics of mandibular bone collagen relative to long bone collagen: relevance to clinical dentistry. BioMed research international, 2014, 769414. https://doi.org/10.1155/2014/769414

Moinzadeh, A. T., Shemesh, H., Neirynck, N. A., Aubert, C., \& Wesselink, P. R. (2013). Bisphosphonates and their clinical implications in endodontic therapy. International endodontic journal, 46(5), 391-398. https://doi.org/10.1111/iej.12018

Moreno-Rabié, C., Gaêta-Araujo, H., Oliveira-Santos, C., Politis, C., \& Jacobs, R. (2020). Early imaging signs of the use of antiresorptive medication and MRONJ: a systematic review. Clinical oral investigations, 24(9), 2973-2989. https://doi.org/10.1007/s00784-020-03423-0

Mourão, C. F. A. B., Moura, A. P., Manso, J. E. F. (2013) Tratamento da osteonecrose dos maxilares associada aos bifosfonatos: revisão da literatura. Rev Bras Cir Cabeça Pescoço, 42(2), 113-117. https://www.researchgate.net/publication/284717613

Ogura I, Minami Y, Ono J, Kanri Y, Okada Y, Igarashi K, Haga-Tsujimura M, Nakahara K, Kobayashi E. CBCT imaging and histopathological characteristics of osteoradionecrosis and medicationrelated osteonecrosis of the jaw. Imaging Sci Dent. 51(1):73- 80. https://doi.org/10.5624/isd.20200230

Ogura I, Minami Y, Ono J, Kanri Y, Okada Y, Igarashi K, Haga-Tsujimura M, Nakahara K, Kobayashi E. CBCT imaging and histopathological characteristics of osteoradionecrosis and medicationrelated osteonecrosis of the jaw. Imaging Sci Dent. 51(1):73-80. https://doi.org/10.5624/isd.20200230

Otto, S., Hafner, S., \& Grötz, K. A. (2009). The role of inferior alveolar nerve involvement in bisphosphonate-related osteonecrosis of the jaw. Journal of oral and maxillofacial surgery: official journal of the American Association of Oral and Maxillofacial Surgeons, 67(3), 589-592. https://doi.org/10.1016/j.joms.2008.09.028

Otto, S., Pautke, C., Hafner, S., Hesse, R., Reichardt, L. F., Mast, G., Ehrenfeld, M., \& Cornelius, C. P. (2013). Pathologic fractures in bisphosphonate-related osteonecrosis of the jaw-review of the literature and review of our own cases. Craniomaxillofacial trauma \& reconstruction, 6(3), 147-154. https://doi.org/10.1055/s-0033-1343776

Papadakis, I., Spanou, A., \& Kalyvas, D. (2021). Success Rate and Safety of Dental Implantology in Patients Treated With Antiresorptive Medication: A Systematic Review. The Journal of oral implantology, 47(2), 169-180. https://doi.org/10.1563/aaid-joi-D-19-00088

Papapoulos, S. E. (2008). Bisphosphonates: how do they work?. Best practice \& research. Clinical endocrinology \& metabolism, 22(5), 831-847. https://doi.org/10.1016/j.beem.2008.07.001

Passeri, L. A., Bértolo, M. B., Abuabara, A. (2011) Osteonecrose dos maxilares associada ao uso de Bifosfonatos. Revista Brasileira de Reumatologia, 51(4), 404-407. SSN: 1809-4570

Pazianas, M., Blumentals, W. A., \& Miller, P. D. (2008). Lack of association between oral bisphosphonates and osteonecrosis using jaw surgery as a surrogate marker. Osteoporosis international: a journal established as result of cooperation between the European Foundation for Osteoporosis and the National Osteoporosis Foundation of the USA, 19(6), 773-779. https://doi.org/10.1007/s00198-007-0547-1

Poubel, V. L. N., Cruz, D. S. M., Gil, L. F., Júnior, N. L. Claus, J. D. P., Gil, J. N. (2012) Osteonecrose maxilo-mandibular induzida por bisfosfonato: revisão bibliográfica. Revista de Cirurgia e TraumatologiaBuco-maxilo-facial, 12(1),33-42. ISSN 1808-5210.

Powell, D., Bowler, C., Roberts, T., Garton, M., Matthews, C., McCall, I., \& Davie, M. (2012). Incidence of serious side effects with intravenous bisphosphonate: a clinical audit. QJM: monthly journal of the Association of Physicians, 105(10), 965-971. https://doi.org/10.1093/qjmed/hcs112 
Querrer, R., Ferrare, N., Melo, N., Stefani, C. M., Dos Reis, P., Mesquita, C., Borges, G. A., Leite, A. F., \& Figueiredo, P. T. (2021). Differences between bisphosphonate-related and denosumab-related osteonecrosis of the jaws: a systematic review. Supportive care in cancer : official journal of the Multinational Association of Supportive Care in Cancer, 29(6), 2811-2820. https://doi.org/10.1007/s00520-020-05855-6

Rasmusson, L., \& Abtahi, J. (2014). Bisphosphonate associated osteonecrosis of the jaw: an update on pathophysiology, risk factors, and treatment. International journal of dentistry, 2014, 471035. https://doi.org/10.1155/2014/471035

Reich, W., Bilkenroth, U., Schubert, J., Wickenhauser, C., \& Eckert, A. W. (2015). Surgical treatment of bisphosphonate-associated osteonecrosis: Prognostic score and long-term results. Journal of cranio-maxillo-facial surger: official publication of the European Association for Cranio-Maxillo-Facial Surgery, 43(9), 1809-1822. https://doi.org/10.1016/j.jcms.2015.07.035

Rigo, R. F., Gomes, F. V., Mayer, L. (2017) Osteonecrose perimplantar relacionada ao uso de bifosfonatos: revisão de literatura. Revista da AcBO, 6(1), 3137 .

Rother, E. . Revisão sistemática X revisão narrativa. Acta paul.enferm., 20(2), v-vi, https://doi.org/10.1590/S0103-21002007000200001

Ruggiero, S. L., \& Woo, S. B. (2008). Biophosphonate-related osteonecrosis of the jaws. Dental clinics of North America, 52(1), 111-ix. https://doi.org/10.1016/j.cden.2007.09.002

Ruggiero, S. L., Dodson, T. B., Assael, L. A., Landesberg, R., Marx, R. E., Mehrotra, B., \& American Association of Oral and Maxillofacial Surgeons (2009). American Association of Oral and Maxillofacial Surgeons position paper on bisphosphonate-related osteonecrosis of the jaws--2009 update. Journal of oral and maxillofacial surgery: official journal of the American Association of Oral and Maxillofacial Surgeons, 67(5 Suppl), 2-12. https://doi.org/10.1016/j.joms.2009.01.009

Ruggiero, S. L., Dodson, T. B., Fantasia, J., Goodday, R., Aghaloo, T., Mehrotra, B., O'Ryan, F., \& American Association of Oral and Maxillofacial Surgeons (2014). American Association of Oral and Maxillofacial Surgeons position paper on medication-related osteonecrosis of the jaw--2014 update. Journal of oral and maxillofacial surgery:fficial journal of the American Association of Oral and Maxillofacial Surgeons, 72(10),1938-1956. https://doi.org/10.1016/j.joms.2014.04.031

Sacco, R., Woolley, J., Yates, J., Calasans-Maia, M. D., Akintola, O., \& Patel, V. (2021). A systematic review of metastatic cancer presenting in osteonecrosis of the jaws (MC-ONJ) in patients undergoing antiresorptive and/or antiangiogenic therapy for skeletal-related adverse events. Oral surgery, oral medicine, oral pathology and oral radiology, 131(6), 650-659. https://doi.org/10.1016/j.0ooo.2021.01.001

Sales, K. O., Conceição, L. S. (2020) A atuação do cirurgião-dentista frente à osteonecrose dos maxilares associada ao uso de bifosfonatos: uma revisão de literatura. Original Article. J Business Techn, 14(2),99-110. ISSN 2526-4281

Santos de Souza, E., de Jesus Santos, J., \& Botelho de Santana, L. L. (2019). Impactos associados ao uso prolongado de bifosfonatos no tratamento da osteoporose em idosos. Revista Textura, 12(20),151-161. https://doi.org/10.22479/desenreg2018v12n20p151-161

Santos, L. C. S., Pereira, R. P., Gusmão, J. M. R., Almeida, O. D. S. (2016). Influência do uso de Bifosfonatos em pacientessubmetidos a implantes dentários: revisão de literatura. Revista Bahiana de Odontologia, Salvador, 7(1), 22-30. http://2238-2720revbahianaodonto.v7i1.706

Santos, P. S. S., Oliveira, M. A., Felix, V. B. (2011) Osteonecrose maxilofacial induzida por Bifosfonatos em indivíduos com osteoporose. Revista Brasileira de Ortopedia,46(5), 495-499, https://doi.org/10.1590/S0102-36162011000500003

Sarasquete, M. E., González, M., San Miguel, J. F., \& García-Sanz, R. (2009). Bisphosphonate-related osteonecrosis: genetic and acquired risk factors. Oral diseases, 15(6), 382-387. https://doi.org/10.1111/j.1601-0825.2009.01568.x

Sawatari, Y., \& Marx, R. E. (2007). Bisphosphonates and bisphosphonate induced osteonecrosis. Oral and maxillofacial surgery clinics of North America, 19(4), 487-vi. https://doi.org/10.1016/j.coms.2007.07.003

Sérgio Perri de Carvalho, P., Fernandes Santos, H., Gomes Duarte, B., Astolphi Carvalho, F., Dias-Ribeiro, E., \& Ferreira Rocha, J. (2010). Principais aspectos da cirurgia bucomaxilofacial no paciente sob terapia com bifosfonatos. Revista Da Faculdade De Odontologia - UPF, 15(2). https://doi.org/10.5335/rfo.v15i2.1351

Sharma, D., Ivanovski, S., Slevin, M., Hamlet, S., Pop, T. S., Brinzaniuc, K., Petcu, E. B., \& Miroiu, R. I. (2013). Bisphosphonate-related osteonecrosis of jaw (BRONJ): diagnostic criteria and possible pathogenic mechanisms of an unexpected anti-angiogenic side effect. Vascular cell, 5(1), 1. https://doi.org/10.1186/2045-824X-5-1

Srinivasan, D., Shetty, S., Ashworth, D., Grew, N., \& Millar, B. (2007). Orofacial pain - a presenting symptom of bisphosphonate associated osteonecrosis of the jaws. British dental journal, 203(2), 91-92. https://doi.org/10.1038/bdj.2007.635

Srivastava, A., Nogueras Gonzalez, G. M., Geng, Y., Won, A. M., Cabanillas, M. E., Naing, A., Myers, J. N., Li, Y., \& Chambers, M. S. (2021). Prevalence of medication related osteonecrosis of the jaw in patients treated with sequential antiresorptive drugs: systematic review and meta-analysis. Supportive care in cancer : official journal of the Multinational Association of Supportive Care in Cancer, 29(5), 2305-2317. https://doi.org/10.1007/s00520-020-05882-3

Stockmann, P., Ebker, T., Bergauer, J., \& Wehrhan, F. (2020). Saliva diagnostics in patients suffering from bisphosphonate-associated osteonecrosis of the jaw: Results of an observational study. Journal of craniomaxillo-facial surgery: official publication of the European Association for Cranio-Maxillo-Facial Surgery, 48(2), 176-180. https://doi.org/10.1016/j.jcms.2020.01.005

Stramandinoli-Zanicotti, R. T., Deliberador, T. M., Schussel, J., Torres-Pereira, C., Sassi, L. M. (2018) Implantes dentários em pacientes usuários de bifosfonatos: o risco de osteonecrose e perda dos implantes é real? Relato de três casos clínicos. RSBO, 15(1),50-9. https://www.researchgate.net/publication/328137190

Tavares Junior, H. H., Almeida, J. S., Mourão, C. F., Meira, R., Ribeiro, J. (2016) Avaliação qualitativa do tratamento da osteonecrose dos maxilares associada aos bifosfonatos: aspectos atuais da literatura. Ciênc Atual, 8(2),02-11. 
Research, Society and Development, v. 10, n. 9, e28410917923, 2021

(CC BY 4.0) | ISSN 2525-3409 | DOI: http://dx.doi.org/10.33448/rsd-v10i9.17923

Tennis, P., Rothman, K. J., Bohn, R. L., Tan, H., Zavras, A., Laskarides, C., Calingaert, B., \& Anthony, M. S. (2012). Incidence of osteonecrosis of the jaw among users of bisphosphonates with selected cancers or osteoporosis. Pharmacoepidemiology and drug safety, 21(8), 810-817. https://doi.org/10.1002/pds.3292

Thumbigere-Math, V., Michalowicz, B. S., Hodges, J. S., Tsai, M. L., Swenson, K. K., Rockwell, L., \& Gopalakrishnan, R. (2014). Periodontal Disease as a Risk Factor for Bisphosphonate-Related Osteonecrosis of the Jaw. Journal of Periodontology, 85(2), 226-233. doi:10.1902/jop.2013.130017

Tsao, C., Darby, I., Ebeling, P. R., Walsh, K., O’Brien-Simpson, N., Reynolds, E., \& Borromeo, G. (2013). Oral Health Risk Factors for BisphosphonateAssociated Jaw Osteonecrosis. Journal of Oral and Maxillofacial Surgery, 71(8), 1360-1366. doi:10.1016/j.joms.2013.02.016

Ulmner, M., Jarnbring, F., \& Törring, O. (2014). Osteonecrosis of the jaw in Sweden associated with the oral use of bisphosphonate. Journal of oral and maxillofacial surgery: official journal of the American Association of Oral and Maxillofacial Surgeons, 72(1), 76-82. https://doi.org/10.1016/j.joms.2013.06.221

Vilela-Carvalho, L. N., Tuany-Duarte., N., Andrade-Figueiredo, M., López-Ortega, K. Osteonecrosis de los maxilares relacionados con el uso de medicamentos: Diagnóstico, tratamiento y prevención. Rev. CES Odont 2018; 31(2), 48-63. http://dx.doi.org/10.21615/cesodon.31.2.5

Walter, C., Laux, C., \& Sagheb, K. (2013). Radiologic bone loss in patients with bisphosphonate-associated osteonecrosis of the jaws: a case-control study. Clinical Oral Investigations, 18(2), 385-390. doi:10.1007/s00784-013-0974-7

Wan, J. T., Sheeley, D. M., Somerman, M. J., \& Lee, J. S. (2020). Mitigating osteonecrosis of the jaw (ONJ) through preventive dental care and understanding of risk factors. Bone research, 8(1), 14. https://doi.org/10.1038/s41413-020-0088-1

Wasserzug, O., Kaffe, I., Lazarovici, T. S., Weissman, T., Yahalom, R., Fliss, D. M., \& Yarom, N. (2017). Involvement of the maxillary sinus in bisphosphonate-related osteonecrosis of the jaw: Radiologic aspects. American journal of rhinology \& allergy, 31(1), 36-39. https://doi.org/10.2500/ajra.2017.31.4395

Zadik, Y., Benoliel, R., Fleissig, Y., \& Casap, N. (2012). Painful trigeminal neuropathy induced by oral bisphosphonate-related osteonecrosis of the jaw: a new etiology for the numb-chin syndrome. Quintessence international (Berlin, Germany : 1985), 43(2), 97-104. PMID: 22257870

Zhang, X., Hamadeh, I. S., Song, S., Katz, J., Moreb, J. S., Langaee, T. Y., Lesko, L. J., \& Gong, Y. (2016). Osteonecrosis of the Jaw in the United States Food and Drug Administration's Adverse Event Reporting System (FAERS). Journal of bone and mineral research : the official journal of the American Society for Bone and Mineral Research, 31(2), 336-340. https://doi.org/10.1002/jbmr.2693 